\title{
Single Dose Inamrinone in Terminal Warm Cardioplegia in On-Pump Coronary Artery Bypass Patients
}

\author{
Aarne Jyrala $^{1 *}$, Nicole M. Gatto ${ }^{2}$, Gregory L. Kay ${ }^{1}$ \\ ${ }^{1}$ Department of Cardiothoracic Surgery, The Heart Institute at Good Samaritan Hospital, Los Angeles, USA; ${ }^{2}$ School of Public \\ Health, Department of Biostatistics, University of California, Los Angeles, USA. \\ Email: *lathoracic@hotmail.com
}

Received December $11^{\text {th }}$, 2011; revised January $28^{\text {th }}$, 2012; accepted February $4^{\text {th }}, 2012$

\begin{abstract}
Background: Phosphodiesterase inhibitors (PDI) are used in cardiac surgery to improve and stabilize cardiac function after surgery. The aim of this study is to evaluate changes in hemodynamics and early outcomes when PDI (Inocor/ inamrinone) is given in terminal warm blood cardioplegia to on-pump CABG only patients and compare results with patients who did not receive the drug. Material and Methods: From April 2003 through September 2004241 pts underwent elective on-pump CABG only surgery. 141 pts received Inocor in the terminal warm blood cardioplegia (Group 1) and 100 pts did not (Group 2). Results: Demographic data, preoperative EuroSCORE risk scores and operative details were similar. Of pts preoperatively in sinus rhythm (SR) 80.15\% in Group 1 and 69.79\% in Group 2 regained spontaneous SR ( $(\mathrm{p}=0.07)$ after release of crossclamp. Inotropic support was needed in 5 pts in Group 1 and in 12 pts in Group 2, p $=0.02$. Post cardiopulmonary by-pass (CPB) IABP support was needed for 4 pts in Group 2 and none for Group 1 pts, $\mathrm{p}$ $=0.01$. There was no operative mortality in either Group and hospital/30 day mortality was similar $(3 / 2.13 \%$ vs $3 / 3.00 \%, p=0.69)$. There were no statistical difference in stay in postoperative intensive care unit $(p=0.15)$, total hospital stay $(p=0.07)$, appearance of postoperative atrial fibrillation $(p=0.23)$ or appearance of postoperative kidney injury $(\mathrm{p}=0.27)$. Post CPB cardiac index improved $16.90 \%$ in Group1but decreased $1.49 \%$ in Group 2, $\mathrm{p}<0.0001$. Mean arterial pressure decreased 7.46\% in Group 1 pts and 5.08\% in Group 2 pts, p $=0.002$, but no pts in either Group needed medication for too low blood pressure. Systemic vascular resistance decreased $28.40 \%$ in Group 1 and $9.02 \%$ in Group 2, p $<0.0001$. Conclusions: Inamrinone in terminal warm blood cardioplegia (hot shot) is safe and effective way to improve and stabilize cardiac function after on-pump CABG surgery but does not affect short-term outcomes.
\end{abstract}

Keywords: Phosphodiesterase Inhibitors; Postoperative Cardiac Function

\section{Introduction}

Phosphodiesterase (PDE) III inhibitors (PDI) are widely used in cardiac surgery [1] to facilitate weaning from cardiopulmonary bypass (CPB) and to stabilize the patient after surgery. Mostly used is Primacor ${ }^{\circledR}$ (milrinone, Sanofi-Aventis, Bridgewater, NJ, USA), which is a derivative of Inocor ${ }^{\circledR}$ (inamrinone, Bedford Laboratories, Bedford, OH, USA) and has several times greater inotropic and vasodilatory properties on weight basis than Inocor but is short-lived (half life approx $30 \mathrm{~min}$ ) and needs initial bolus dose and continued intravenous administration which often leads to low systemic blood pressure and subsequent need for drugs to increase blood pressure $[2,3]$ Inocor has a half-life of 3.6 hours and is able to sustain its effect for the critical early postoperative hours when given in a single dose before or after release of cross-

${ }^{*}$ Corresponding author. clamp [4-7]. The hemodynamic effects of both drugs are similar [8].

We hypothesized that giving a PDI directly into the coronaries rather than intravenously or into CPB would result in better cardiac recovery and performance after CPB and possibly have less vasodilatory effects than systemic delivery. Intracoronary administration has been studied with CHF patients and shown to be effective in increasing cardiac performance acutely [9]. There are no earlier reports available about intracoronary use of PDI's in combination with cardiac surgery. The PDI was given preemptively to all pts operated with cardiopulmonary by-pass by two surgeons. One surgeon did not give PDI to any patient, so we were able to compare two groups of elective on-pump CABG surgery patients for differences which may be achieved by giving preemptive Inocor intracoronary with the hot shot.

The aim of this study is to evaluate the safety and effects of intracoronary administration of Inocor after elec- 
tive on-pump CABG surgery. The effects are compared with a matching population of patients who did not receive Inocor.

The study was approved by the hospital's research committee.

\section{Material and Methods}

From April 2003 through September 2004510 pts underwent on-pump cardiac surgery at our institute. Only elective on-pump CABG patients were included into the study. Patients with concomitant cardiac procedures, valve or aortic procedures, emergency operations, patients with ongoing inotrope infusions and pts with preoperative IABP support were excluded leaving 241 pts for the study.

At the time of the study two surgeons gave preemptive Inocor to every pt and one surgeon did not give it to any pt and did not contribute in the preparation of manuscript. This created two groups of pts: Inocor group (Group 1) $n=$ 141 pts and no Inocor group (Group 2) n = 100 pts.

25 - $50 \mathrm{mg}$ Inocor was given into the terminal warm cardioplegia (hot shot) by perfusionist. The dose was dependent on the size of the patient. Pts with a BSA $>2 \mathrm{~m}^{2}$ were given $50 \mathrm{mg}$ and smaller pts $25 \mathrm{mg}$. The dose is about two thirds of the manufacturer recommended starting dose.

Cardiac output, cardiac index (CI), arterial pressure were measured and systemic vascular resistance (SVR) calculated when pts were anesthetized but before opening the chest and again 30 - 45 minutes after cross-clamp had been released and the patient weaned from CPB. The results were documented on the anesthesia sheet. Mean arterial pressures (MAP) were calculated using formula $\mathrm{MAP}=($ Syst BP $+2 \times$ Diast BP $) / 3$. Operative and perfusion reports were analyzed for number of grafts, length of $\mathrm{CPB}$, length of aortic cross-clamp, need for cathecholamines at the time of separation from CPB and need for IABP support. Spontaneous return of sinus rhythm (SR) of pts preoperatively in SR were documented.

Early outcomes [stay in the postoperative intensive care unit (ICU)], length of hospitalization (LOS), occurrence of postoperative atrial fibrillation (AF) and acute kidney injury (AKI), operative and hospital/30 day mortality) were obtained from hospital charts of all pts.

\section{Statistical Analysis}

Variables were examined in their continuous form and categorized as follows: baseline serum creatinine $(>2.2$ $\mathrm{mg} / \mathrm{dl},<2.25 \mathrm{mg} / \mathrm{dl}$ ) and left ventricular ejection fraction $(<0.3,>0.3)$. Demographic characteristics, EuroSCORE variables, operative factors and short-term follow-up measures were summarized by administration of PDI in the hot shot (Group 1-Inocor $n=141$, Group 2-no Inocor, $\mathrm{n}=100$ ). Variables were compared between Groups using simple parametric t-test or chi $=$ square test and $\mathrm{a}$ p-value $<0.05$ was regarded as statistically significant.

All analyses used SAS version 9.2 (SAS Institute Inc. Cary, NC, USA).

\section{Surgical Technique}

All pts were operated through standard median sternotomy. Ascending aorta was cannulated when feasible and single venous cannulation with vacuum assistance was used for venous return. Moderate hypothermia to $34^{\circ} \mathrm{C}$ was used during CPB.

Cold whole blood cardioplegia was used given antegradely and retrogradely. Retrograde cardioplegia was given continuously during cross-clamp time stopping only when it interfered with visualization and for maximum of 10 minutes at a time. Pts were rewarmed in the later stages of the procedure and when the pts temperature was $>36^{\circ} \mathrm{C}, 500 \mathrm{ml}$ of warm blood was given retrogradely (hot shot). Inocor 25 - $50 \mathrm{mg}$ was added to this by the perfusionist to Group 1 pts. Cross-clamp was removed when the hot shot was completed.

Anesthesia was conducted according to hospital approved protocol in all cases.

\section{Results}

The demographic variables and ES risk scores were equal between the groups and no single variable had a statisticcally significant difference. The data is summarized in Tables 1 and 2.

Comparing operative details the number of grafts, $\mathrm{CPB}$ and cross-clamp times were equal. Appearance of spontaneous sinus rhythm in pts in preoperative SR after release of cross-clamp was quite similar between the Groups: Group 1 pts $(109 / 80.15 \%$ vs 67/69.79\%, p = 0.07). Need for cathecholamines for weaning and stabilization the patient was lower in Group 1 pts (5/3.55\% vs 12/12.00\%, $\mathrm{P}=0.01)$. No pt needed IABP support after CPB in Group 1 but 4 pts in Group 2, p = 0.01 .

Postoperative outcomes did not differ statistically although there was a tendency for longer ICU and LOS and higher incidence of postoperative AF in Group 2 pts. There were no difference in appearance of AKI (defined as postoperative rise in s-crea $>2.2 \mathrm{mg} / \mathrm{dl}$ ). Outcomes are summarized in Table 3.

Mean pre CPB CI, MAP and SVR did not differ between the groups. CI improved after CPB in Group 1 with $16.90 \%, \mathrm{p}<0.0001$, while in Group 2 CI decreased by $1.49 \%, \mathrm{p}=0.64$.

MAP decreased significantly in both groups, $7.46 \%$ vs $4.82 \%, \mathrm{p}<0.0001$ for Group 1 pts and $\mathrm{p}=0.002$ for Group 2 pts. There was no pt in either Group who needed drugs for too low blood pressure. 
Table 1. Demographics of Groups 1 and 2 patients.

\begin{tabular}{llll}
\hline Variable & $\begin{array}{l}\text { Group } 1, \mathrm{n}=141 \\
\mathrm{~m} \pm \mathrm{SD} / \mathrm{n}(\%)\end{array}$ & $\begin{array}{l}\text { Group 2, } \mathrm{n}=100 \\
\mathrm{~m} \pm \mathrm{SD} / \mathrm{n}(\%)\end{array}$ & $\mathrm{p}$ \\
\hline Age, years & $66.76 \pm 9.56$ & $66.31 \pm 11.36$ & 0.71 \\
Females & $50(35.50)$ & $32(32.00)$ & 0.58 \\
NYHA & $2.71 \pm 0.62$ & $2.78 \pm 0.76$ & 0.48 \\
LVEF & $0.48 \pm 0.14$ & $0.48 \pm 0.14$ & 0.93 \\
LVEF $<0.3$ & $11(7.81)$ & $13(13.00)$ & 0.19 \\
CHF & $34(24.11)$ & $22(22.00)$ & 0.76 \\
DIABETES & $53(37.58)$ & $49(49.00)$ & 0.08 \\
PTS IN PREOP SR & $136(96.45)$ & $96(96.00)$ & 0.86 \\
S-CREA & $1.27 \pm 1.03$ & $1.28 \pm 1.04$ & 0.96 \\
S-CREA $>2.2$ mg/dl & $7(4.96)$ & $6(6.00)$ & 0.73 \\
RE-DO & $14(9.93)$ & $10(10.00 \%)$ & 0.99 \\
3VD & $78(67.38)$ & $68(68.00)$ & 1.00 \\
2VD & $36(25,53)$ & $18(18.00)$ & 0.21 \\
1VD & $10(7.09)$ & $14(14.00)$ & 0.09 \\
LM $>$ 70\% & $31(21.99)$ & $13(13.00)$ & 0.09 \\
AES & $5.48 \pm 3.31$ & $5.23 \pm 3.26$ & 0.57 \\
LES & $7.80 \pm 10.02$ & $7.44 \pm 9.08$ & 0.77 \\
\hline
\end{tabular}

NYHA: New York Heart Association functional classification; LVEF: left ventricular ejection fraction; CHF: congestive heart failure; SR: sinus rhythm; S-CREA: serum creatinine concentration in mg/dl; RE-DO: reoperation on the heart; 3VD: triple vessel coronary artery disease; 2VD: double vessel disease; 1VD: single vessel disease; LM: left main coronary artery stenosis; AES: additive EuroSCORE points; LES: logistic EuroSCORE \%.

Table 2. Operative details.

\begin{tabular}{llll}
\hline Variable & $\begin{array}{l}\text { Group 1 } \\
\mathrm{m} \pm \mathrm{SD} / \mathrm{n}(\%)\end{array}$ & $\begin{array}{l}\text { Group 2 } \\
\mathrm{m} \pm \mathrm{SD} / \mathrm{n}(\%)\end{array}$ & $\mathrm{p}$ \\
\hline Grafts/patient & $3.51 \pm 0.99$ & $3.52 \pm 1.11$ & 0.92 \\
$\mathrm{CPB}$ & $121.72 \pm 34.71$ & $115.90 \pm 34.78$ & 0.20 \\
X-clamp & $102.50 \pm 28.72$ & $95.27 \pm 27.20$ & 0.05 \\
Spont SR & $109(80.15)$ & $67(69.79)$ & 0.07 \\
Inotropes $^{1}$ & $5(3.55)$ & $12(12.00)$ & 0.01 \\
IABP support & 0 & $4(4.00)$ & 0.01 \\
Oper. mortality & 0 & 0 & \\
\hline
\end{tabular}

CPB: cardiopulmonary bypass time, minutes; X-clamp: aortic cross clamp time, minutes; SR: sinus rhythm; IABP: intra-aortic balloon pulsation device; ${ }^{1}$ Cathecolamines, dopamine, adrenaline or noradrenaline for weaning off $\mathrm{CPB}$ and continued to intensive care unit.

Table 3. Postoperative outcomes.

\begin{tabular}{llll}
\hline Variable & $\begin{array}{l}\text { Group 1 } \\
\mathrm{m} \pm \mathrm{SD} / \mathrm{n}(\%)\end{array}$ & $\begin{array}{l}\text { Group 2 } \\
\mathrm{m} \pm \mathrm{SD} / \mathrm{n}(\%)\end{array}$ & $\mathrm{p}$ \\
\hline Hospital/30 day mortality & $3(2.13)$ & $3(3.00)$ & 0.67 \\
ICU days & $1.53 \pm 1.66$ & $2.12 \pm 4.38$ & 0.15 \\
LOS days & $7.79 \pm 5.43$ & $9.16 \pm 6.24$ & 0.08 \\
Postop AF & $13(10.74)$ & $16(16.67)$ & 0.11 \\
Postop $\mathrm{AKI}^{2}$ & $11(8.21)$ & $12(12.77)$ & 0.28 \\
\hline
\end{tabular}

ICU: stay in postoperative intensive care unit; LOS: total hospital stay; AF: atrial fibrillation; AKI: acute kidney injury, postoperative rise in s-crea $>2.2 \mathrm{mg} / \mathrm{dl}$. ${ }^{1} \mathrm{Of}$ pts with preoperative SR, Group $1 \mathrm{n}=136$, Group $2 \mathrm{n}=94$; ${ }^{2}$ Of pts with preoperative s-crea $<2.25 \mathrm{mg} / \mathrm{dl}$, Group $1 \mathrm{n}=133$ Group 2, n = 94 . 
SVR decreased in both Groups after CPB, significantly in Group, 28.40\% 1, p $<0.0001$ but not significantly in Group 2 9.02\%, $p=0.05$. See Table 4.

\section{Comments}

PDE III inhibitors were discovered in the 70's and their hemodynamic effects soon evaluated [10] which led to widespread use in cardiac surgery and used instead or together with cathecholamines for pts who developed low output after CPB [11-13]. Several different dosing regimens have been used usually with a loading dose and iv-infusion for several hours post surgery. Single dosage use has been tried specifically with Inocor $[4,6,7]$ with its longer half life. The drugs are usually given into the CPB or intravenously with no obvious hemodynamic differences but both leaves the heart for second or third pass of the drug. Also the timing of introducing the drug has many modifications, they have been given prior to $\mathrm{CPB}$, at the end of $\mathrm{CPB}$ or after СРB but all regimens seem to have good hemodynamic effects.

The heart is the primary target for PDI but there are no clinical reports available where the drug have been given into coronary circulation (i.e. via cardioplegia) at the end or CPB. There is one recent experimental study from Ko and coworkers from 2009 [5] where high dose of Inocor was given in the terminal warm blood cardioplegia before releasing the crossclamp. They observed much improved cardiac function with Inocor vs no Inocor specifically increased myocardial cAMP. Coronary perfusion was also higher with Inocor as reported for humans also [14]. They concluded that (one dose) Inocor promote a rapid and sustained cardiac functional recovery with various cellular protective effects $[15,16]$ after open heart surgery Our idea was to deliver Inocor directly into the heart in order to have the first pass of the drug in the heart/myocardium and possibly have less effect of the vasodilatory properties with less use of medications to boost low blood pressure commonly seen with intravenous infusions.

Our results parallel the results of Ko [5] although we do not have any biochemical data. Comparing the groups there is a definite improvement in cardiac performance in Group 1 specially comparing pre- and post CPB CI and less use of cathecolamine support.

SVR and MAP also decreased statistically more in Group 1 but there were no instances where the blood pressure needed to be medically improved. This is in contrast to giving PDI as a loading dose and continue with intravenous infusion and it has been recommended to give simultaneous norepinephrine with this dosing. Intracardiac route decreases or eliminates the inappropriate fall of blood pressure but is highly effective in promoting better hemodynamics and stability in the early postoperative hours.

There are no statistical differences in early outcomes as reported by others $[17,18]$ although there is a tendency of longer ICU and LOS in Group 2 pts.

The reason for postoperative AF is multifactorial but theoretically good myocardial recovery together with good myocardial protection should result in lesser amount of postoperative AF but it was not evident in this study. In a recent report Fleming and coworkers [19] found increased amount of postoperative AF when PDI was used after separation from CPB but the pts who had AF had lower EF, were older, had longer CPB times and already had cathecholamine support which all result in an increase in postoperative AF.

No adverse effects of Inocor were observed.

\section{Drawbacks}

There are several shortcomings in this study. It is an observational retrospective single unit study with rather low numbers of patients.

The goal of this study was to analyze hemodynamic effects and safety of a not earlier reported way of delivering preemptive PDI during on-pump CABG surgery.

\section{Conclusions}

Preemptive intracardiac/intracoronary PDI at the end of CPB is safe and reliable way to improve hemodynamics and myocardial recovery after cardiac surgery.

Table 4. Changes in cardiac index, mean arterial pressure and systemic vascular resistance post CPB.

\begin{tabular}{llll}
\hline Variable & $\begin{array}{l}\text { Group 1 } \\
\mathrm{m} \pm \mathrm{SD} / \mathrm{n}(\%)\end{array}$ & $\begin{array}{l}\text { Group 2 } \\
\mathrm{m} \pm \mathrm{SD} / \mathrm{n}(\%)\end{array}$ & $\mathrm{p}$ \\
\hline Pre CPB CI & $2.61 \pm 0.66$ & $2.68 \pm 0.56$ & 0.35 \\
Post CPB CI & $3.03 \pm 0.74$ & $2.64 \pm 0.58$ & $<0.0001$ \\
Pre CPB MAP & $83.60 \pm 8.17$ & $83.64 \pm 8.17$ & 0.97 \\
Post CPB MAP & $77.36 \pm 5.12$ & $79.61 \pm 5.87$ & 0.002 \\
Pre CPB SVR & $1277.16 \pm 421.54$ & $1277.50 \pm 390.55$ & 0.99 \\
POST CPB SVR & $914.48 \pm 278.50$ & $1162.214 \pm 413.64$ & $<0.0001$ \\
\hline
\end{tabular}

CPB: cardiopulmonary bypass; CI: cardiac index, $1 / \mathrm{min} / \mathrm{m}^{2}$; MAP: mean arterial pressure, mmHg; SVR: systemic vascular resistance, dyne* $\mathrm{sec} / \mathrm{cm}^{5}$. 
When given directly into the heart with terminal warm cardioplegia the expected improvements in hemodynamics are prominent but the strong vasodilatation seen with intravenous use is decreased.

The study was funded by Elma Larsson Foundation and Los Angeles Thoracic and Cardiovascular Foundation. Los Angeles, USA.

\section{REFERENCES}

[1] M. Kikura and S. Sato, "The Efficacy of Preemptive Milrinone or Amrinone Therapy in Patients Undergoing Coronary Artery Bypass Grafting,” Anesthesia \& Analgesia, Vol. 94, No. 1, 2002, pp. 22-30.

[2] J.-F. Hardy, N. Searic, M. Roy and J. Perrault, “Amrinone, in Combination with Norepinephrine, Is an Effective First-Line Drug for Difficult Separation from Cardiopulmonary Bypass," Canadian Journal of Anesthesia, Vol. 40, No. 6, 1993, pp. 495-501.

[3] T. Yamada, J. Takeda, N. Katori, K. Tsuzaki and R. Ochial, "Hemodynamic Effects of Milrinone during Weaning from Cardiopulmonary Bypass: Comparison of Patients with a Low and High Prebypass Cardiac Index," Journal of Cardiothoracic and Vascular Anesthesia, Vol. 14, No. 4, 2000, pp. 367-373. doi:10.1053/jcan.2000.7920

[4] Y. Hamada, K. Kawachi, T. Yamamoto, T. Nakata, Y. Kashu, M. Sato and Y. Watanabe, "Effects of single administration of a phophodiesterase III inhibitor during cardiopulmonary bypass. Comparison of Milrinone and Amrinone,” Japanese Circulation Journal, Vol. 63, No. 8, 1999, pp. 605-6095. doi:10.1253/jcj.63.605

[5] Y. Ko, K. Morita, R. Nagahori, K. Kinouchi, G. Shinohara, H. Kagawa and H. Hashimoto, "Myocardial Cyclic AMP Augmentation with High-Dose PDE III Inhibitor in Terminal Warm Blood Cardioplegia,” Annals of Thoracic and Cardiovascular Surgery, Vol. 15, No. 5, 2009, pp. 311-316.

[6] K. J. Fogg and D. Royston, "Improved Performance with Single Dose Phosphodiesterase Inhibitor (Editorial),” British Journal of Anaesthesia, Vol. 81, No. 5, 1998, pp. 663-666.

[7] M. Kikura, J. H. Levy, J. M. Bailey, J. S. Shanewise, L. G. Michelsen and S. M. Sadel, "A Bolus Dose of $1.5 \mathrm{mg} / \mathrm{kg}$ Amrinone Effectively Improves Low Cardiac Output State Following Separation from Cardiopulmonary Bypass in Cardiac Surgical Patients," Acta Anaesthesiologica Scandinavica, Vol. 42, No. 7, 1998, pp. 825-833. doi:10.1111/j.1399-6576.1998.tb05329.x

[8] J. P. Rathmell, R. C. Prielipp, J. F. Butterworth, E. Williams, F. Villamaria, L. Testa, C. Viscomi, F. P. Ittleman, E. C. Baisden and L. R. Royster, "A Multicenter, Randomized, Blind Comparison of Amrinone with Milrinone after Elective Cardiac Surgery,” Anesthesia \& Analgesia, Vol. 86, 1998, pp. 683-690.

[9] P. L. Ludmer, R. F. Wright, J. M. Arnold, P. Ganz, E. Braunwald and W. S. Colucci, "Separation of the Direct Myocardial and Vasodilator Actions of Milrinone Ad- ministered by Intracoronary Infusion Technique,” Circulation, Vol. 73, No. 1, 1986, pp. 130-137.

doi:10.1161/01.CIR.73.1.130

[10] R. J. Benotti, W. Grossman, E. Braunwald, D. D. Davolos and A. A. Alousi, "Hemodynamic Assessment of Amrinone,” New England Journal of Medicine, Vol. 299, 1978, pp. 1373-1377. doi:10.1056/NEJM197812212992501

[11] K. P. Lewis, I. R. Appadurai, E. T. Pierce, E. F. Halpern and R. H. Bode Jr., "Prophylactic Amrinone for Weaning from Cardiopulmonary Bypass,” Anaesthesia, Vol. 55, No. 7, 2000, pp. 627-633.

doi:10.1046/j.1365-2044.2000.01372.x

[12] J. F. Butterworth, R. L. Royster, R. C. Prielipp, S. T. Lawless and S. L. Wallenhaupt, "Amrinone in Cardiac Surgical Patients with Left Ventricular Dysfunction. A Prospective, Randomized Placeo-Controlled Trial," Chest, Vol. 104, No. 6, 1993, pp. 1660-1667. doi:10.1378/chest.104.6.1660

[13] M. Gillies, R. Bellomo, L. Doolan and B. Buxton, "Benchto-Bedside Review: Inotropic Drug Therapy after Adult Cardiac Surgery-Systematic Literature Review,” Critical Care, Vol. 9, No. 3, 2005, pp. 266-279.

[14] M. Arbeus, B. Axelsson, O. Friberg, A. Magnuson, L. Bodin and J. Hultman, "Milrinone Increases Flow in Coronary Artery Bypass Grafts after Cardiopulmonary Bypass: A Prospective, Randomized, Double-Blind, Placebo-Controlled Study,” Journal of Cardiothoracic and Vascular Anesthesia, Vol. 23, No. 1, 2009, pp. 48-53. doi:10.1053/j.jvca.2008.07.005

[15] E. B. Lobato, F. Urdaneta, T. D. Martin and N. Gravenstein, "Effects of Milrinone versus Epinephrine on Grafted Internal Mammary Artery Flow after Cardiopulmonary Bypass," Journal of Cardiothoracic and Vascular Anesthesia, Vol. 14, No. 1, 2000, pp. 9-11. doi:10.1016/S1053-0770(00)90047-8

[16] V. Simkova, P. Radermacher and E. Barth, "Metabolic Effects of Phosphodiesterase III Inhibitors: Another Reason to Promote Their Use?” Critical Care, Vol. 11, 2007, pp. 139-140. doi:10.1186/cc5924

[17] M. Jebeli, M. Ghazinoor, M. H. Mandegar, M. R. Rasouli, P. Eghtesadi-Araghi, H. Goodarzynejad, R. Mohaamadzadeh, A. Darehzereshki and S. Dianat, "Effect of Milrinone on Short-Term Outcome of Patients with Myocardial Dysfunction Undergoing Coronary Artery Bypass Graft: A Randomized Controlled Trial,” Cardiology Journal, Vol. 17, No. 1, 2010, pp. 73-78.

[18] M. Heringlake, M. Wernerus, J. Grünfeld, S. Klaus, H. Heinze, M. Bechtel, L. Bahlmann, J. Poeling and J. Schön, "The Metabolic and Renal Effects of Adrenaline and Milrinone in Patients with Myocardial Dysfunction after Coronary Artery Bypass,” Critical Care, Vol. 11, No. 2, 2007, pp. R51-R61. doi:10.1186/cc5904

[19] G. A. Fleming, K. T. Murray, C. Yu, J. G. Byrne, J. P. Greelish, M. R. Petracek, S. J. Hoff, S. K. Ball, N. J. Brown and M. Pretorius, "Milrinone Use Is Associated with Postoperative Atrial Fibrillation after Cardiac Surgery," Circulation, Vol. 118, No. 16, 2008, pp. 16191625. doi:10.1161/CIRCULATIONAHA.108.790162 